\title{
Minimization of Shadow Effects in Forest Canopies for Chlorophyll Content Estimation Using Red Edge Optical Indices through Radiative Transfer: Implications for MERIS
}

\author{
P. J. Zarco-Tejada ${ }^{1,3}$, and J. R. Miller ${ }^{2}$ \\ ${ }^{1}$ Centre for Research in Earth and Space Science (CRESS), York University, Toronto, Ontario M3J 1P3, Canada \\ ${ }^{2}$ Dept. of Physics and Astronomy, York University \& Centre for Research in Earth and Space Technology (CRESTech), \\ 4700 Keele Street, Toronto, Ontario M3J 1P3, Canada \\ ${ }^{3}$ Now at Center for Spatial Technologies and Remote Sensing (CSTARS), Department of Land, Air, and Water \\ Resources (LAWR), University of California, Davis CA 95616-8671 USA - pzarco@ucdavis.edu
}

\begin{abstract}
This paper reports on progress made to develop methods to accurately estimate pigment content in forest canopies from airborne hyperspectral data. Radiative transfer approaches and red edge optical indices were applied to twelve sites of Acer saccharum $M$. in the Algoma Region, Ontario (Canada), where field measurements and hyperspectral CASI reflectance data have been collected between 1997 and 2000 deployments. Turbid-medium models such as SAILH and Kuusk were successfully applied to closed forest canopies minimizing the effects of canopy shadows and structure by using red edge optical indices in the merit function. Pigment content was successfully estimated linking PROSPECT leaf model to SAILH canopy model, minimizing the merit function by iterative process in MERIS-derived spectra from hyperspectral CASI data. The selection of the merit function used for minimization has been demonstrated to be critical, showing that shadow effects are removed when red edge optical indices are used. Implications for MERIS sensor by ESA on ENVISAT are discussed in this paper.
\end{abstract}

\section{INTRODUCTION}

The estimation of pigment content at a canopy level can be performed using simple statistical relationships at a leaf level through the use of optical indices, using modelling methods through radiative transfer by numerical model inversion [1]-[6], and by a combination of leaf-level empirical relationships coupled with a canopy reflectance (CR) model [7],[8]. Coupling leaf and canopy models for biochemical and biophysical parameter estimation by model inversion enables to consider the type of vegetation canopy and viewing geometry. CR models, such as SAILH [9] and MCRM [10],[11] used in this research, take into account viewing geometry and canopy structure, therefore modelling those effects in the canopy reflectance by different approximations generally based on the radiative transfer equation and geometrical optical considerations. Less complex assumptions of such turbid-medium models make its application to remote sensing easier since the number of input variables to be minimized decreases, although they lack the modelling of canopy structural variables that affect reflectance, such as canopy shadows and openings. The numerical model inversion of linked leaf and canopy models requires a merit function $\Delta^{2}$ for a set of parameters $P=\left(N, C h l_{a+b}, C_{w}, L A I, \theta_{s} \ldots\right)$ that minimizes the error between the modelled and sensor-measured canopy reflectance. The effect of selecting the merit function for biochemical and biophysical parameter estimation is demonstrated to be critical [12]. The effect of shadows and openings in the canopy reflectance for pigment content estimation using hyperspectral CASI data of $2 \mathrm{~m}$ spatial resolution and MERIS-simulated data is studied in this paper, and the implications for MERIS sensor discussed.

\section{DATA COLLECTION}

Airborne hyperspectral data acquisition was carried out with $0.5 \mathrm{~m}$ spatial resolution and 5 spectral bands, and with $2 \mathrm{~m}$ spatial resolution and 72 channels in the 400$950 \mathrm{~nm}$ spectral range. Leaf samples were collected at each site for biochemical analysis and measurement of leaf chlorophyll $a+b$ and carotenoid concentrations. Single leaf reflectance and transmittance measurements were acquired on leaf samples using a Li-Cor 1800 Sphere apparatus with a fibre spectrometer with $7.5 \mathrm{~nm}$ spectral resolution in the $400-900 \mathrm{~nm}$ range. Radiometrically calibrated CASI data were processed to ground-reflectance using the CAM5S atmospheric correction model with aerosol optical depth data collected in the study area at the time of data acquisition. Reflectance data were georeferenced using GPS data collected onboard the aircraft.

\section{MERIT FUNCTION DEFINITION FOR THE RADIATIVE TRANSFER MODEL INVERSION}

Numerical model inversion of a linked leaf and canopy model to estimate chlorophyll $a+b$ requires minimizing a 
merit function by iterative optimization. The merit function $\Delta^{2}=\sum_{\mathrm{n}}\left[\rho_{\mathrm{m}}\left(\lambda_{\mathrm{i}}\right)-\rho^{*}\left(\lambda_{\mathrm{i}}, \mathrm{P}\right)\right]^{2}$ is used for error calculation when optimizing the set of parameters input for the leaf and canopy reflectance models. The measured canopy spectral reflectance $\rho_{m}\left(\lambda_{i}\right)$ is compared to $\rho^{*}\left(\lambda_{i}, P\right)$, the modelled canopy spectral reflectance calculated with a set of $P$ parameters $P=\left(N, C h l_{a+b}, C_{w}, L A I, \theta_{s} \ldots\right)$. The critical role played by the merit function used for parameter estimation has been treated before [12],[13] showing that red edge spectral transforms such as $\mathrm{R}_{750} / \mathrm{R}_{710}$ in the merit function minimize the effects due to forest canopy structure, shadows and openings in the measured canopy reflectance. Therefore this approach enables the use of less complicated radiative transfer models, such as turbid medium assumptions, for closed forest canopies.

\section{RESULTS AND IMPLICATIONS FOR MERIS}

Results from 1997, 1998 and 1999 data over 12 study sites of Acer saccharum M. for $\mathrm{chl}_{\mathrm{a}+\mathrm{b}}$ estimation using the spectral transform $\mathrm{R}_{750} / \mathrm{R}_{710}$ in the merit function showed that little effect is caused by shadows in the estimation of $\mathrm{chl}_{\mathrm{a}+\mathrm{b}}$ when the red edge optical index $\mathrm{R}_{750} / \mathrm{R}_{710}$ is used in the merit function: RMSE $=5.57 \mu \mathrm{g} / \mathrm{cm}^{2}$ (targeting crowns, $2 \times 2 \mathrm{~m}$ pixel size), $\mathrm{RMSE}=5.48 \mu \mathrm{g} / \mathrm{cm}^{2}$ (all pixels including shadows, $20 \times 20 \mathrm{~m}$ plot) with SAILH and PROSPECT inversion. A large effect is found when all reflectance channels are used in the minimizing function: $\mathrm{RMSE}=12 \mu \mathrm{g} / \mathrm{cm}^{2}$ (targeting crowns), RMSE $=23.1 \mu \mathrm{g} / \mathrm{cm}^{2}$ (all pixels) [12],[13]. A validation of this methodology was carried out selecting a different set of $1480 \times 80 \mathrm{~m}$ plots collected at $0.86 \times 3.4 \mathrm{~m}$ and re-sampled to $1.5 \times 1.5 \mathrm{~m}$ spatial resolution. This validation enabled us to propose an operational methodology for $\mathrm{chl}_{\mathrm{a}+\mathrm{b}}$ estimation in closed canopies using a satellite sensor with red edge spectral bands, such as MERIS by ESA, and to study its spectral capabilities for model inversion. MERIS equivalent spectra were calculated from the CASI-72-channel data in order to perform a simulation to study the applicability of MERIS red edge bands for pigment estimation by model inversion. Although careful atmospheric correction must be performed, two MERIS spectral bands located along the red edge, at $705 \mathrm{~nm}$ (10nm bandwidth) and $753.75 \mathrm{~nm}$ (7.5 $\mathrm{nm}$ bandwidth) are proposed for calculating the index $\mathrm{R}_{750} / \mathrm{R}_{705}$ to be used in the merit function. The similar spectral bandwidth of the CASI sensor in the 72-channel mode of operation of $7.5 \mathrm{~nm}$, allowed simulation of the MERIS centre wavelengths by interpolation from CASI hyperspectral data. MERIS-equivalent images were built from CASI data, and reflectance data extracted from the plots (Figure 1). The same methodologies were then carried out for $\mathrm{chl}_{\mathrm{a}+\mathrm{b}}$ estimation by model inversion using SAILH and $\mathrm{R}_{\infty 3}$ infinite reflectance model [12] coupled with PROSPECT, using MERIS $\mathrm{R}_{750} / \mathrm{R}_{705}$ red edge index. Results in Table 1 show that $R_{750} / R_{705}$ used for MERIS performs similarly as $R_{750} / R_{710}$ used for estimations with CASI, obtaining $\mathrm{R}^{2}=0.44$, and RMSE $=3.0 \mu \mathrm{g} / \mathrm{cm}^{2}$ (SAILH + PROSPECT, targeting crowns), RMSE $=3.97 \mu \mathrm{g} / \mathrm{cm}^{2}$ (SAILH+PROSPECT, all pixels).

\section{CONCLUSIONS}

This work demonstrates that red edge optical indices used in the merit function for model inversion minimizes effects due to shadows and openings in forest canopies. This method enables the use of less complex radiative transfer turbid-medium models for pigment content estimation. Results show only a small effect when shadow pixels are included for the estimation of the $\mathrm{chl}_{\mathrm{a}+\mathrm{b}}$ using SAILH and PROSPECT inversion with the red edge optical index $\mathrm{R}_{750} / \mathrm{R}_{710}$ in the merit function. On the other hand, a large effect due to inclusion of shadow pixels is found with SAILH and PROSPECT inversion when all reflectance channels are used in the minimizing function. MERISsimulated data from hyperspectral CASI reflectance showed that MERIS index $\mathrm{R}_{750} / \mathrm{R}_{705}$ achieved RMSE of 3 $\mu \mathrm{g} / \mathrm{cm}^{2}$ using PROSPECT+SAILH by model inversion. These results suggest that methodologies investigated here using red edge indices can be transferred to MERIS

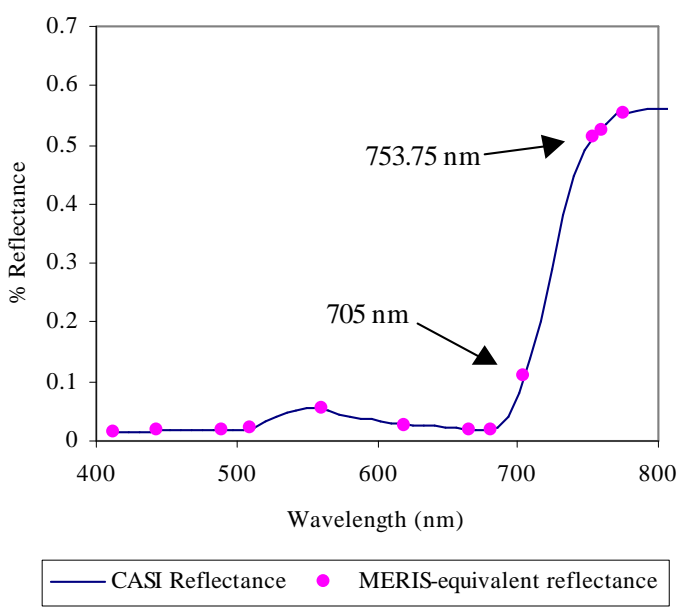

Figure 1. MERIS-equivalent spectra obtained from CASI 72-channel data showing the spectral bands proposed for model inversion through the red edge optical index $\mathrm{R}_{750} / \mathrm{R}_{705}$. 
Table 1. Comparison of RMSE and $\mathrm{R}^{2}$ for $\mathrm{chl}_{\mathrm{a}+\mathrm{b}}$ estimation considering all pixels (including shadows) in the $80 \times 80 \mathrm{~m}$ area averaged reflectance of $0.86 \times 3.4 \mathrm{~m}$ resampled to $1.5 \times 1.5 \mathrm{~m}$ spatial resolution and 72 -channel CASI data (2809 pixels), and selecting the upper $25 \%$ pixels in the NIR to minimize shadows and openings in a dense canopy of Acer saccharum M. (targeting crowns). MERIS-equivalent spectra were obtained from CASI 72 -channel data, and $\mathrm{R}_{750} / \mathrm{R}_{705}$ used for model inversion with SAILH and $\mathrm{R}_{\infty 3}$ infinite reflectance coupled with PROSPECT.

\begin{tabular}{|c|c|c|c|c|c|c|}
\hline & \multicolumn{6}{|c|}{ CASI 72-channel data } \\
\hline & \multicolumn{2}{|c|}{$\begin{array}{c}\text { SAILH+ } \\
\text { PROSPECT } \\
\Delta^{2}=f\left(\Sigma \mathbf{R}_{\lambda}\right)\end{array}$} & \multicolumn{2}{|c|}{$\begin{array}{c}\text { SAILH+ } \\
\text { PROSPECT } \\
\Delta^{2}=f\left(\mathbf{R}_{750} / \mathbf{R}_{710}\right)\end{array}$} & \multicolumn{2}{|c|}{$\begin{array}{c}\mathbf{R}_{\infty 3}+ \\
\text { PROSPECT } \\
\Delta^{2}=f\left(\mathbf{R}_{750} / \mathbf{R}_{710}\right)\end{array}$} \\
\hline & $u p$ & all & up & all & up & all \\
\hline $\mathrm{R}^{2}$ & 0.2 & 0.18 & 0.42 & 0.43 & 0.43 & 0.44 \\
\hline \multirow[t]{4}{*}{ RMSE } & 4.8 & 14.8 & 3.0 & 5.2 & 5.0 & 8.9 \\
\hline & \multicolumn{6}{|c|}{ MERIS Simulation from CASI 72-channel data } \\
\hline & \multicolumn{3}{|c|}{$\begin{array}{c}\text { SAILH+ } \\
\text { PROSPECT } \\
\Delta^{2}=f\left(\mathbf{R}_{750} / \mathbf{R}_{705}\right)\end{array}$} & \multicolumn{3}{|c|}{$\begin{array}{c}\mathbf{R}_{\infty 3}+\text { PROSPECT } \\
\Delta^{2}=f\left(\mathbf{R}_{750} / \mathbf{R}_{705}\right)\end{array}$} \\
\hline & $U p$ & & all & & & all \\
\hline $\mathrm{R}^{2}$ & $\overline{0.42}$ & & 0.44 & & & 0.43 \\
\hline RMSE & 3.0 & & 3.97 & & & 6.98 \\
\hline
\end{tabular}

\section{REFERENCES}

[1] S. Jacquemoud, "Inversion of the PROSPECT+SAIL canopy reflectance model from AVIRIS equivalent spectra: theoretical study", Remote Sensing of Environment, 44:281-292, 1993.

[2] S. Jacquemoud, F. Baret, B. Andrieu, F.M. Danson, and K. Jaggard, "Extraction of vegetation biophysical parameters by inversion of the PROSPECT+SAIL models on sugar beet canopy reflectance data. Application to TM and AVIRIS sensors", Remote Sensing of Environment, 52:163172, 1995.

[3] A. Kuusk, "Monitoring of vegetation parameters on large areas by the inversion of a canopy reflectance model", International Journal of Remote Sensing, 19:2893-2905, 1998.

[4] V. Demarez, and J.P. Gastellu-Etchegorry, "A Modeling Approach for Studying Forest Chlorophyll Content", Remote Sensing of Environment, 71:226-238, 2000.
[5] M. Weiss, F. Baret, R.B. Myneni, A. Pragnere, and Y. Knyazikhin, "Investigation of a model inversion technique to estimate canopy biophysical variables from spectral and directional reflectance data", Agronomie, 20:3-22, 2000.

[6] S. Jacquemoud, C. Bacour, H. Poilve, and J.P. Frangi, "Comparison of four radiative transfer models to simulate plant canopies reflectanceDirect and inverse mode", Remote Sensing of Environment,74:471-481, 2000.

[7] P.J. Zarco-Tejada, J.R. Miller, G.H. Mohammed, T.L. Noland, and P.H. Sampson, "Chlorophyll Fluorescence Effects on Vegetation Apparent Reflectance: II. Laboratory and Airborne CanopyLevel Measurements with Hyperspectral data", Remote Sensing of Environment, 74(3), 2000.

[8] P.J. Zarco-Tejada, "Hyperspectral Remote Sensing of Closed Forest Canopies: Estimation of Chlorophyll Fluorescence and Pigment Content", Ph.D. Dissertation, Graduate Program in Earth and Space Science, York University, Toronto, September, 2000.

[9] W. Verhoef, "Light scattering by leaf layers with application to canopy reflectance modelling: the SAIL model", Remote Sensing of Environment, 16:125-141, 1984.

[10] A. Kuusk, "A Markov chain model of canopy reflectance", Agricultural and Forest Meteorology, 76:221-236, 1995. "A computer-efficient plant canopy reflectance model", Computers \& Geosciences, 22:149-163, 1996.

[12] P.J. Zarco-Tejada, J.R. Miller, T.L. Noland, G.H. Mohammed, and P.H. Sampson, Scaling-up and model inversion methods with narrow-band optical indices for chlorophyll content estimation in closed forest canopies with hyperspectral data, IEEE Transactions on Geosciences and Remote Sensing, (in press).

[13] P.J. Zarco-Tejada, Hyperspectral remote sensing of closed forest canopies: Estimation of chlorophyll fluorescence and pigment content, Ph.D. Dissertation, Graduate Program in Earth and Space Science, York University, Toronto, 2000. 